\title{
SÍNTESE E CARACTERIZAÇÃO DE NANOESFERAS DE POLIESTIRENO PARA ENCAPSULAMENTO DE NANOPARTICULADO MAGNÉTICO
}

\section{SYNTHESIS AND CHARACTERIZATION OF POLYSTYRENE NANOESPHERES FOR MAGNETIC NANOPARTICULATE ENCAPSULATION}

\author{
G. G. M. ELYSEU ${ }^{1}$, R. V. FERREIRA ${ }^{2}$ e M. R. DUMONT ${ }^{2}$ \\ ${ }^{1}$ Universidade Federal de Minas Gerais, Departamento de Química, Brasil \\ ${ }^{2}$ Centro Federal de Educação Tecnológica de Minas Gerais, Departamento de Engenharia de \\ Materiais, Brasil \\ gmelyseu@gmail.com
}

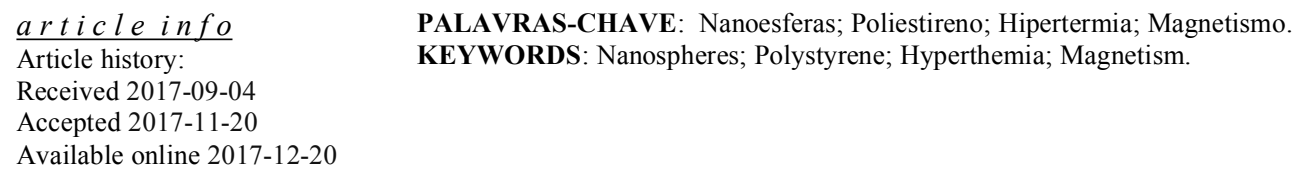

RESUMO: Nanopartículas com propriedades magnéticas podem atuar no combate oncológico por meio da hipertermia terapêutica a qual provoca a desnaturação de proteínas e, portanto, o enfraquecimento de células tumorais. A principal vantagem desta técnica é efeitos colaterais mais brandos do que os tratamentos convencionais. Objetivando uma ativação indesejada do sistema imune este trabalho propõe desenvolver uma rota para a obtenção de partículas nanométricas de poliestireno (PS) visando encapsular tais partículas. Foi utilizada uma rota livre de emulsificantes o que garantiria uma maior pureza do sistema. O material sintetizado foi analisado através microscopia eletrônica de varredura, granulometria por difração a laser, análise térmica e espectroscopia no infravermelho. Foi confirmada a obtenção de poliestireno no formato esférico com tamanho médio de partícula de $317 \mathrm{~nm} \pm 58 \mathrm{~nm}$ adequada para o uso em sistemas biológicos.

\begin{abstract}
Nanoparticles with magnetic properties can be used in cancer treatment through thermotherapy which promotes protein denaturation and, therefore, damaging cancer cells. The main advantage of this technique is milder collateral effects than conventional treatments. To avoid undesired activation of the immune system this work proposes to develop a route to obtain nanoparticles of polystyrene (PS) in order to encapsulate such particles. Emulsifiers free route was used which would guarantee a higher purity of the system. The synthesized material was analyzed by scanning electron microscopy, laser diffraction granulometry, thermal analysis and infrared spectroscopy. Spherical polystyrene with an average particle size of $317 \mathrm{~nm} \pm 58 \mathrm{~nm}$, suitable for use in biological systems, was confirmed.
\end{abstract}

\section{INTRODUÇÃO}

A partir da década passada, nanomateriais à base de polímeros passaram a ser amplamente estudados devido às inúmeras aplicações em diferentes áreas. Dentre os polímeros descritos na literatura, destaca-se a importância do poliestireno (PS) que, devido à sua fácil manipulação, é usado como modelo para estudos de sistemas mais complexos (HE, PAN, 
2004). Outra propriedade interessante dessa classe de materiais é a possibilidade serem produzidos com estruturas ocas o que atribui uma série de características promissoras, como, uma maior área superficial e menor densidade. Isto também permite o transporte de partículas no seu interior conferindo a eles a possibilidade de transporte controlado de substâncias, o que os torna recursos interessantes para as áreas biomédicas (NANDIYANTO et al., 2012). A partir desse princípio, iniciou-se a uma nova área de pesquisa em nanotecnologia de compósitos, uma vez que, além de substratos orgânicos, complexos inorgânicos também podem ser encapsulados. Assim, a partir desses estudos, têm sido catalogados novos materiais de alto desempenho (LANDJ et al., 2013).

De modo geral, os sistemas clássicos de estudo da física e da química não são aplicáveis à nanotecnologia. Deste modo, as propriedades de um material em escalas macrométricas não se repetirão em escalas nanométricas, por exemplo, o atrito não age da mesma forma em diferentes escalas (ALEXIS et al. 2008). Portanto, materiais nanométricos a apresentam propriedades químicas, físicas e biológica devido a efeitos quânticos (BHATTACHARYA; MUKHERJEE, 2010).

O desenvolvimento da nanotecnologia implica avanços científicos de outras áreas do conhecimento, como por exemplo, engenharia e áreas da saúde. Segundo Farokzad e Langer (2006), atribui-se particular interesse à área médica, que até o presente momento, tem a oncologia como área com o maior potencial de melhoramento, evidenciado pelos investimentos governamentais em pesquisas. O governo americano investiu em $2004 \mathrm{em}$ pesquisas nanotecnológicas em seis áreas relacionadas ao câncer, entre elas o carreamento controlado de fármacos por meio de nanoesferas (ALEXIS et al. 2008). Mesmo com o esforço para o desenvolvimento da área, muito pouco é conhecido sobre a interação entre o nanocompósito e os sistemas biológicos. Por exemplo, biomateriais são susceptíveis a mudança de suas superfícies quando em contato com biomoléculas, o que exige um maior estudo da área. Demanda particular esforço o estudo da biocompatibilidade se torna complexo e depende de inúmeras variáveis como tamanho, forma, superfície e revestimento, topografia, carga, aglomeração, concentração, entre outros (LOU et al. 2010). Logo, torna-se claro a necessidade e importância do estudo da casca polimérica que envolverá o substrato inorgânico ou orgânico (LANDJ et al., 2013).

\subsection{Tratamento Oncológico: Hipertermia}

Quando se fala sobre o aumento da expectativa de vida é impossível não associar ao desenvolvimento da biomedicina como vetor do combate às doenças. Dentre as patologias com maior incidência na população mundial está o câncer, segunda maior causa de óbitos no mundo. Mesmo com o avanço da ciência, não se tem a garantia de haver um tratamento completamente eficiente contra a doença (SIEGEL et al., 2012). Devido à grande variedade de tipos de câncer e a especificidade do combate a cada um deles, existem diversas técnicas aplicada no tratamento oncológico, por exemplo, quimioterapia, radiação e cirurgia (GIUSTINI et al., 2010). Todas essas técnicas possuem vantagens e desvantagens associadas aos recursos empregados, porém, destas se destaca a falta de seletividade das drogas aplicadas em tratamentos contra câncer específicos. Uma grande dificuldade enfrentada é a manutenção de uma taxa ótima de concentração do fármaco no local do tratamento, que tem como solução prática a utilização de altas doses de fármaco, o que acarreta em maiores 
efeitos colaterais. Uma alternativa à manutenção da eficiência das técnicas citadas com uma menor dose de fármaco é a aplicação da nanopartículas encapsuladas (MAHMOUDI et al., 2011). Segundo Misra et al., (2010), o recurso da nanotecnologia, nesse cenário, é importante pois é possível manipular a concentração desejada de fármacos na região tumoral, o que coibiria o câncer com o menor prejuízo às células sadias. Essa característica especialmente útil quando combinado com uma das terapias padrões empregados no mercado (HUANG et al., 2011).

No desenvolvimento da nanotecnologia em combate oncológico surgiu também um novo procedimento: o tratamento via hipertermia (MAHMOUDI et al., 2011). A hipertermia é o tratamento oncológico que tem como recurso o aquecimento localizado de tumores malignos até uma temperatura ótima de aproximadamente $43{ }^{\circ} \mathrm{C}$ por um período de 30 minutos (SHUBAYEV et al., 2009). Quando associada à nanotecnologia essa técnica se mostra particularmente eficiente na indução da apoptose ou enfraquecimento de células tumorais. Isso se dá em função de nanopartículas magnéticas, que, devido à alternância do campo magnético, consegue aquecer até a temperatura desejada (GIUSTINI et al., 2010).

\subsection{Nanoparticulas Magnéticas}

Atualmente são poucos os materiais com atividade magnética que cumprem os requisitos para serem imerso em um sistema biológico. O óxido de ferro se destaca devido à sua biocompatibilidade comparado a outros compostos inorgânicos (BOURGEAT-LAMI, LANG 1998). Dentre os inúmeros compostos assumidos pelo óxido de ferro destaca-se a magnetita $\left(\mathrm{Fe}_{3} \mathrm{O}_{4}\right)$, que possui um momento magnético suficiente, estabilidade química em meio fisiológico e baixa toxicidade, sendo um bom candidato para atuar como agente de um tratamento de hipertermia (MAHMOUDI et al., 2011; FIGUEROLA et al. 2010). Quando revestidas de poliestireno (PS), as propriedades de superfície das nanopartículas magnéticas são preservadas reduzindo no sistema a formação de aglomerações, mudanças na geometria molecular, nem degradação devido a exposição ao meio fisiológico (MAHMOUDI et al., 2011; RAMOS, FORCADA, 2011). Além disso a baixa distribuição de tamanho e forma final que podem ser obtidas na partícula revestida de PS será importante para o tratamento médico (FARIDI-MAJIDI et al. 2006). al., 2011).

\subsection{Síntese de Nanoesfera de Poliestireno livre de emulsificante}

Existem inúmeras pesquisas que descrevem diferentes rotas de sínteses para as nanoesferas de PS e também para o encapsulamento no seu interior (NANDIYANTO et al., 2012; LADJ et al., 2013). As técnicas mais recorrentes na literatura são: emulsão, miniemulsão, microemulsão e síntese livre de emulsificantes. Esse processo livre de emulsificantes prevê um sistema dotado de duas fazer imiscíveis: água - fase polar - e o monômero de estireno fase apolar. Ao se colocar o sistema sob forte agitação constante o monômero será subdividido em várias gotículas nanométricas, dando origem a uma suspensão coloidal estável sob agitação (LANDFESTER, 2009). Para dar origem às nanoesferas, utiliza-se um iniciador de polimerização, normalmente inorgânico no sistema (NANDIYANTO et al., 2012), que poderá ser solúvel em qualquer uma das duas fases. Uma das características fundamentais de materiais nanométricos é a grande área superficial assegurando que as colisões entre o iniciador e a gotícula são favorecidas. Assim sendo, cada uma das gotículas 
se torna uma fonte de polimerização do sistema, passando então do estado líquido para o sólido (LANDFESTER, 2009).

$\mathrm{Na}$ síntese livre de emulsificantes existem diversas vantagens em relação aos demais processos. Primeiramente é possível separar o produto final por sedimentação com auxílio de uma centrífuga, pois, não existem outras substâncias no meio, atingindo um alto índice de pureza no produto final (MACHADO et al., 2007). Em segundo lugar a síntese livre de emulsificante possui facilidade de separação por faixa de tamanho (ALEXIS et al., 2008; LANDFESTER, 2009). A biocinética postula que o tamanho adequado para nanoparticulas in vivo devem estar entre $10 \mathrm{~nm}$ e $200 \mathrm{~nm}$. Maiores que $10 \mathrm{~nm}$ para que escapem da depuração plasmática, e menores que $200 \mathrm{~nm}$ para não ativar a resposta imune do sistema reticuloendotelial do pâncreas e do fígado (SHUBAYEV et al., 2009). Finalmente não é necessária a manutenção da fase contínua do monômero para a obtenção de resultados satisfatórios (SHUBAYEV et al., 2009; MACHADO et al., 2007). São descritas na literatura as mais diversas nanopartículas que podem ser encapsuladas por essa técnica: $\mathrm{TiO}_{2}, \mathrm{CaCO}_{3}$, $\mathrm{ZnO}, \mathrm{Fe}_{3} \mathrm{O}_{4}$ (LANDFESTER, 2009).

\subsection{Formação do Nanocompósito: Encapsulamento}

Algumas nanopartículas inorgânicas podem ativar facilmente o sistema imune causando respostas indesejadas, portanto, é indiscutível a necessidade do encapsulamento com material inerte (LADJA, R. et al.,2013). Para que ocorra o encapsulamento, pode-se por exemplo, fazer uma mistura prévia entre o monômero de estireno e o material inorgânico. Deste modo ao adicionar no sistema aquoso o iniciador de polimerização o processo ocorreria como descrito anteriormente (LANDFESTER, 2009).

\section{MATERIAL E MÉTODOS}

\subsection{Materiais}

As esferas de poliestireno foram obtidas através do monômero de estireno $99 \% \mathrm{C}_{8} \mathrm{H}_{8}$ e o persulfato de potássio $99 \% \mathrm{~K}_{2} \mathrm{~S}_{2} \mathrm{O}_{8}$ adquiridos da Sigma-Aldrich, hidróxido de sódio $\mathrm{NaOH}$ $99 \%$ adquirido da Merck e 2-propanol 99,9\% $\mathrm{C}_{3} \mathrm{H}_{8} \mathrm{O}$ da Synth. A água deionizada ultra pura foi obtida por sistema Mili-q da Milipore Corporation. Todos os reagentes foram obtidos em grau analítico e utilizados sem tratamentos prévios.

\subsection{Síntese de nanoesferas de PS livre de emulsificante}

Seguindo o modelo proposto por Loiola e colaboradores (2008), uma alíquota de $210 \mathrm{ml}$ de monômero de estireno foi lavada, para retirada do estabilizante 4-tert-Butylcatechol, com $200 \mathrm{ml}$ de solução, previamente preparada, de $\mathrm{NaOH} 0,1 \mathrm{~mol} / \mathrm{L}$ em funil de separação e o processo foi repetido por três vezes. Em sequência a amostra foi lavada com água ultrapura, de modo a retirar o resíduo da solução de $\mathrm{NaOH}$ e novamente o procedimento foi repetido por três vezes. Em um balão de 3 pescoços foi adicionado $140 \mathrm{ml}$ de água ultrapura. Foi conectado, em uma das entradas, um condensador para evitar a evaporação dos solventes. Outra entrada recebeu uma rolha móvel com termômetro. E à terceira entrada foi conectado um tubo com fluxo de nitrogênio gasoso continuo para manter a atmosfera inerte durante todo processo de síntese. O sistema foi mantido sob agitação constante em temperatura de 60 
${ }^{\circ} \mathrm{C}$ (agitador magnético com aquecimento IKA; HS 7) por 60 minutos para a remoção completa do oxigênio dissolvido na água. Uma alíquota de $16 \mathrm{ml}$ do monômero de estireno lavado foi separada e aquecida, de modo a não criar um ponto frio na solução, em um béquer paralelamente à purga do sistema com água. Para conclusão dessa etapa, o estireno foi então adicionado à solução e a agitação foi aumentada para cerca de $1500 \mathrm{rpm}$. O sistema foi mantido nessas condições por 60 minutos. Foi preparada uma solução contendo 0,053 g de persulfato de potássio em $10 \mathrm{ml}$ de água destilada. Esta solução, antes de ser versada no sistema, foi aquecida para que não representasse um ponto frio. $\mathrm{O}$ iniciador foi vertido lentamente e a solução final foi mantida sob agitação. O sistema foi recoberto por papel alumínio para diminuir a troca de calor com o meio externo e a síntese se manteve sob essas condições por 24 horas.

\subsection{Separação das Nanoesferas de Poliestireno}

A suspensão coloidal de poliestireno obtida foi diluída, na proporção volumétrica de 1:9, com isopropanol para facilitar a separação das partículas com a água. A solução foi centrifugada por 3 horas em rotação de $1200 \mathrm{rpm}$. No fim desse processo o precipitado foi recolhido, em placa de Petri, e submetido a procedimento de secagem em temperatura ambiente por 24 horas.

\subsection{Caracterização}

Amostras do precipitado foram recolhidos com uma fita de carbono e em seguida metalizada com ouro, para tornar condutora a superfície do material, e analisadas no equipamento de Microscopia Eletrônica de Varredura (MEV) Shimadzu, SSX-550. A análise Espectroscopia de Infravermelho por Transformada de Fourier (FTIR) (Shimadzu, IR-Prestige 21) foi realizada no modo ATR de $400 \mathrm{~cm}^{-1}$ a $4000 \mathrm{~cm}^{-1}$. A análise Termogravimétrica (Shimadzu, DTG-60H) foi feita a partir de $10,825 \mathrm{mg}$ de amostra, colocados em um cadinho de alumina sob um fluxo constante de nitrogênio de $50 \mathrm{ml} / \mathrm{min}$. Alíquotas da suspenção obtida antes da centrifugação foram dispersadas em água, com agitação mecânica e ultrassom por 60 segundos até obscuração de $12 \%$, e analisadas por granulometria por difração a laser (Cilas, 1090).

\section{RESULTADOS E DISCUSSÕES}

\subsection{Síntese}

Nos primeiros minutos de síntese, após a adição do iniciador, a suspensão adquiriu um aspecto branco leitoso, o que segundo Loiola e colaboradores (2008) seria indicador da reação de polimerização. Findado o experimento, a suspensão de nanoesferas de PS foi armazenada e se manteve estável por até 30 dias após a síntese. Ao final da lavagem e secagem das nanoesferas de PS, foi obtido um pó fino branco.

\subsection{Microscopia Eletrônica de Varredura}

O resultado obtido ao MEV é demonstrado na Figura 1. 

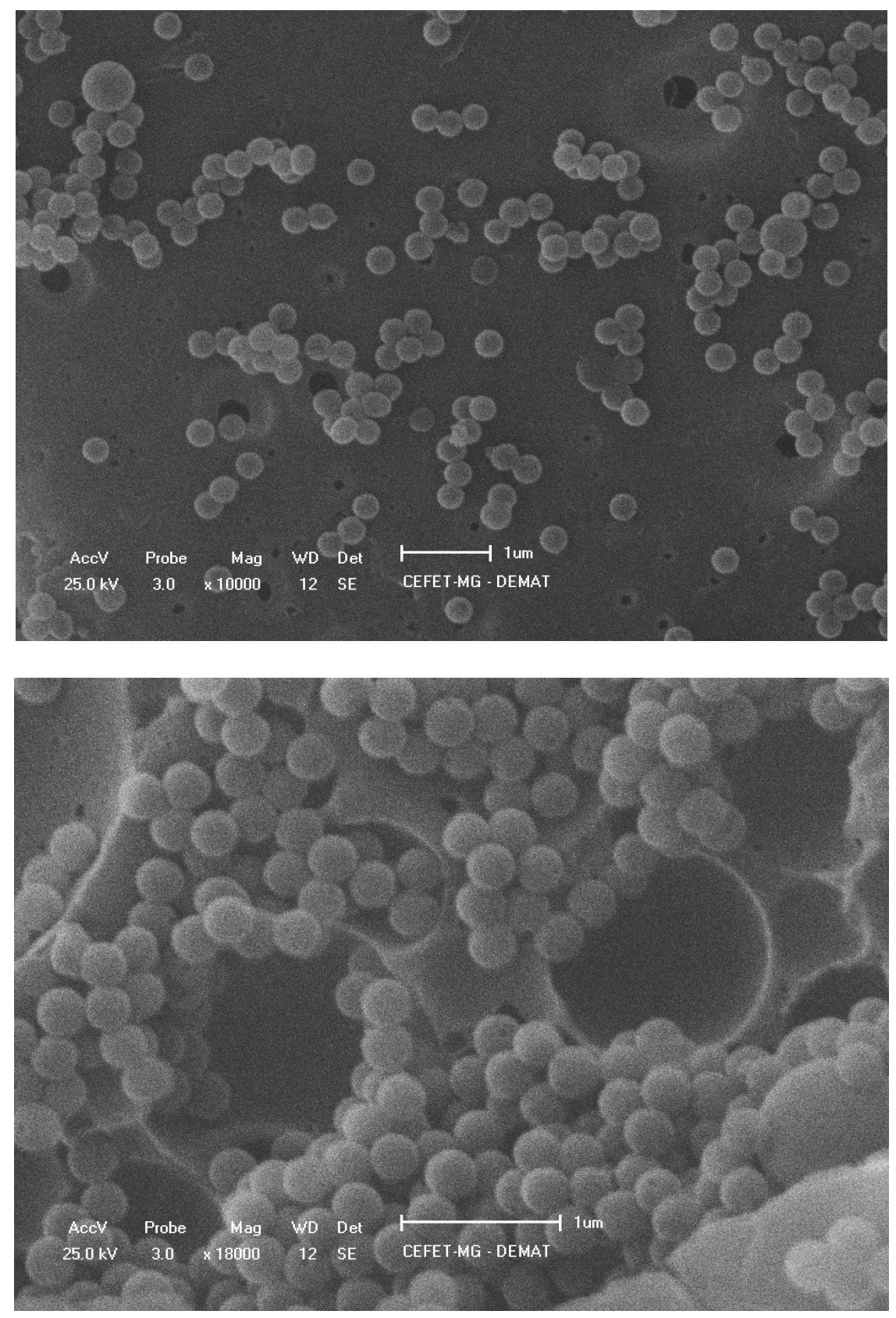

Figura 1 - Imagens obtidas ao MEV das nanoesferas de poliestireno em diferentes ampliações.

O material obtido apresenta formato esférico, como previsto, com pequena dispersão de tamanho. Foram obtidas seis imagens aleatórias ao MEV e dez nanoesferas de cada foto foram mensuradas. Foram medidas sessenta esferas com tamanhos entre $177 \mathrm{~nm}$ e $456 \mathrm{~nm}$ e a média encontrada foi de $317 \mathrm{~nm} \pm 58 \mathrm{~nm}$.

Diferentemente da bibliografia consultada, as nanoesferas estão inseridas em uma faixa de dispersão menor do que as descritas. Segundo Dumont (2016), ao seguir o mesmo processo 
foram encontradas nanoesferas entre $68 \mathrm{~nm}$ e $968 \mathrm{~nm}$, enquanto Sem e colaboradores (2004) encontraram uma variação entrem $230 \mathrm{~nm}$ e $857 \mathrm{~nm}$. Se, por um lado, o espaço amostral foi menor, é fácil perceber que as nanoesferas têm tamanhos homogêneos nas micrografias apresentadas. Ainda segundo Dumont (2016), foram encontradas duas faixas de tamanho para as esferas, entre $300 \mathrm{~nm}$ e $400 \mathrm{~nm}$ e entre $800 \mathrm{~nm}$ e $900 \mathrm{~nm}$. Entretanto, ao analisar as imagens obtidas ao MEV, é possível observar apenas uma faixa de dispersão.

\subsection{Infravermelho}

O resultado obtido na espectroscopia de infravermelho está ilustrado na Figura 2.

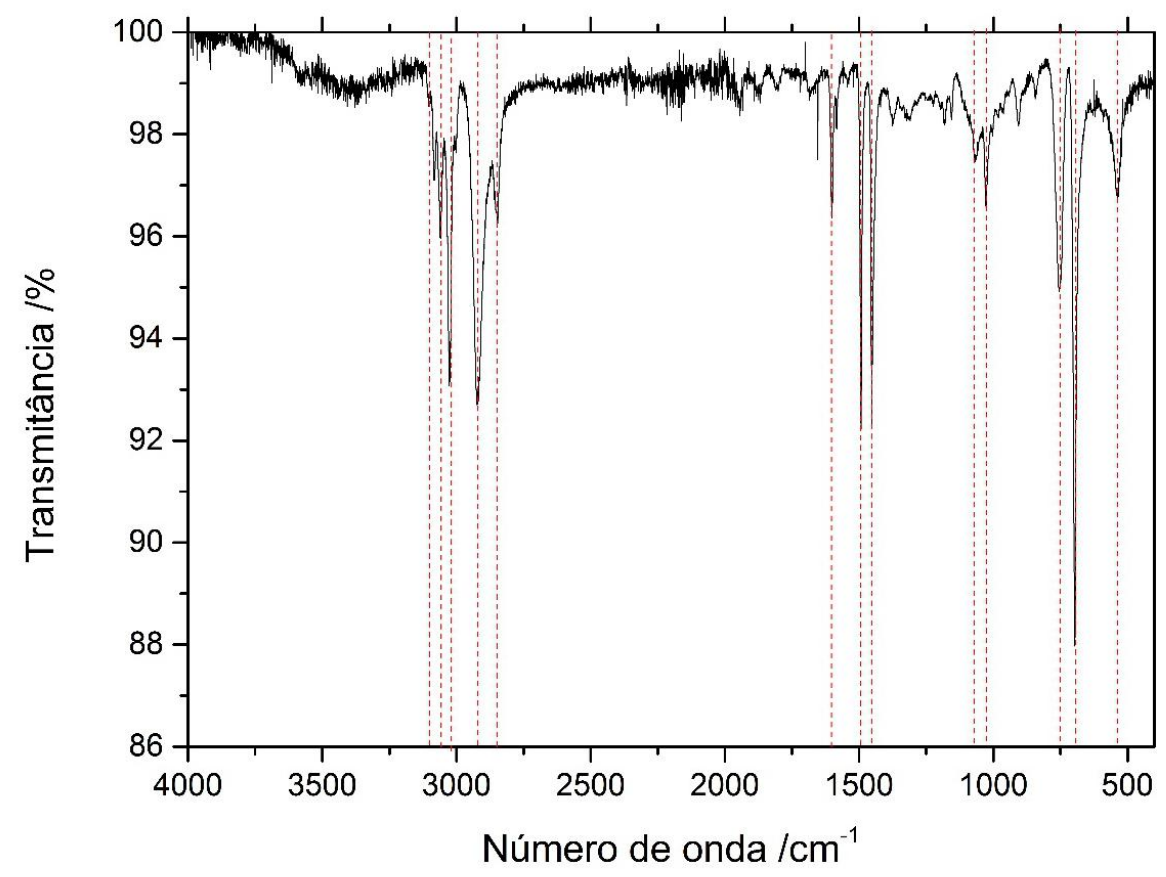

Figura 2 - Espectro de infravermelho obtido para a caracterização de nanoesferas de poliestireno

As principais bandas encontradas para o material analisado permitem caracterizá-lo como poliestireno Liang (1958) e Wibawa (2011) e estão resumidas na Tabela 1, com o modo vibracional associado. O Infravermelho para o poliestireno convencional já é descrito há algumas décadas e, segundo Liang e Krimm (1958), tradicionalmente é estudado entre 500 $\mathrm{cm}^{-1}$ e $3300 \mathrm{~cm}^{-1}$. As bandas principais descritas na literatura para o poliestireno são: 3030 $\mathrm{cm}^{-1}$ a $3100 \mathrm{~cm}^{-1}$ referente ao estiramento $v \mathrm{C}-\mathrm{H}$ fenílico; $1475 \mathrm{~cm}^{-1}$ a $1625 \mathrm{~cm}^{-1}$ indicam o estiramento $\vee \mathrm{C}=\mathrm{C}-\mathrm{C}$ conjugado; $650 \mathrm{~cm}^{-1}$ a $900 \mathrm{~cm}^{-1}$ indicam a vibração $v$ fenílica (WIBAWA, 2011). 
Tabela 1 - Atribuições das principais bandas

\begin{tabular}{cc}
\hline Número de Onda $\left(\mathrm{cm}^{-1}\right)$ & Modo Vibracional associado \\
\hline 3100 & $v$ C-H fenílico \\
3056 & $v$ C-H fenílico \\
3020 & $v$ C-H fenílico \\
2920 & $v$ H-C-H fenílico \\
2848 & $v$ H-C-H fenílico \\
1601 & $v$ C $=$ C-C conjugado \\
1494 & $v$ C $=$ C-C conjugado \\
1443 & $\delta$ C-H fenílico \\
1069 & $\delta$ C-H fenílico \\
1026 & $\delta$ C-H fenílico \\
752 & $v$ fenílico \\
694 & $v$ fenílico
\end{tabular}

\subsection{Análise termogravimétrica}

O resultado obtido para a análise termogravimétrica está ilustrado na Figura 3.

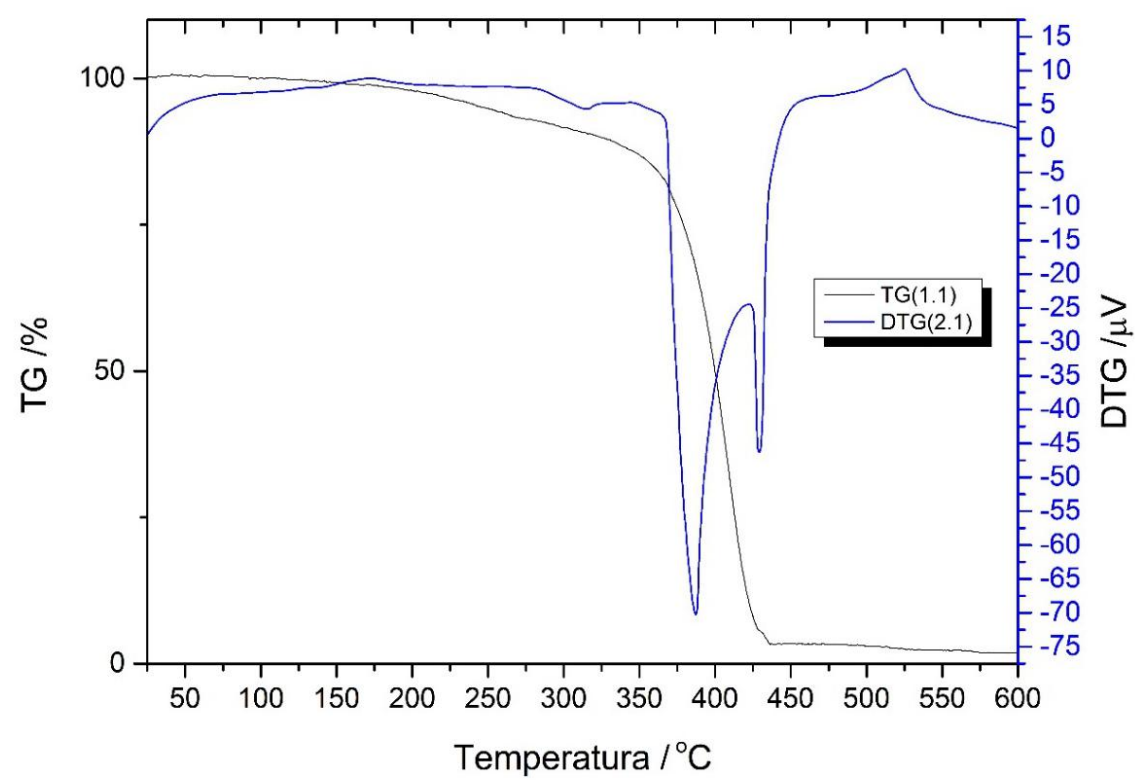

Figura 3 - TG e DTG obtido para a caracterização de nanoesferas de poliestireno 
O poliestireno apresentou perda de $2 \%$ em massa a partir de $100{ }^{\circ} \mathrm{C}$ até $388^{\circ} \mathrm{C}$ e esta perda possivelmente está associada à perda de água presente no sistema. A partir de $388{ }^{\circ} \mathrm{C}$ ocorre a degradação das cadeias poliméricas até aproximadamente $427{ }^{\circ} \mathrm{C}$, temperatura na qual praticamente todo o material já foi consumido. Esses resultados estão congruentes com os encontrados por Liang e Krimm (1958), Rieger (1996) e Nandiyato (2013).

\subsection{Granulometria por Difração a Laser}

Para esse ensaio, foi separada uma alíquota de $40 \mathrm{ml}$ da suspensão de nanoesferas que foi versada no equipamento até que atingisse o valor recomendado e foi feita uma leitura de acordo com o tamanho das partículas que é apresentado na Figura 4.

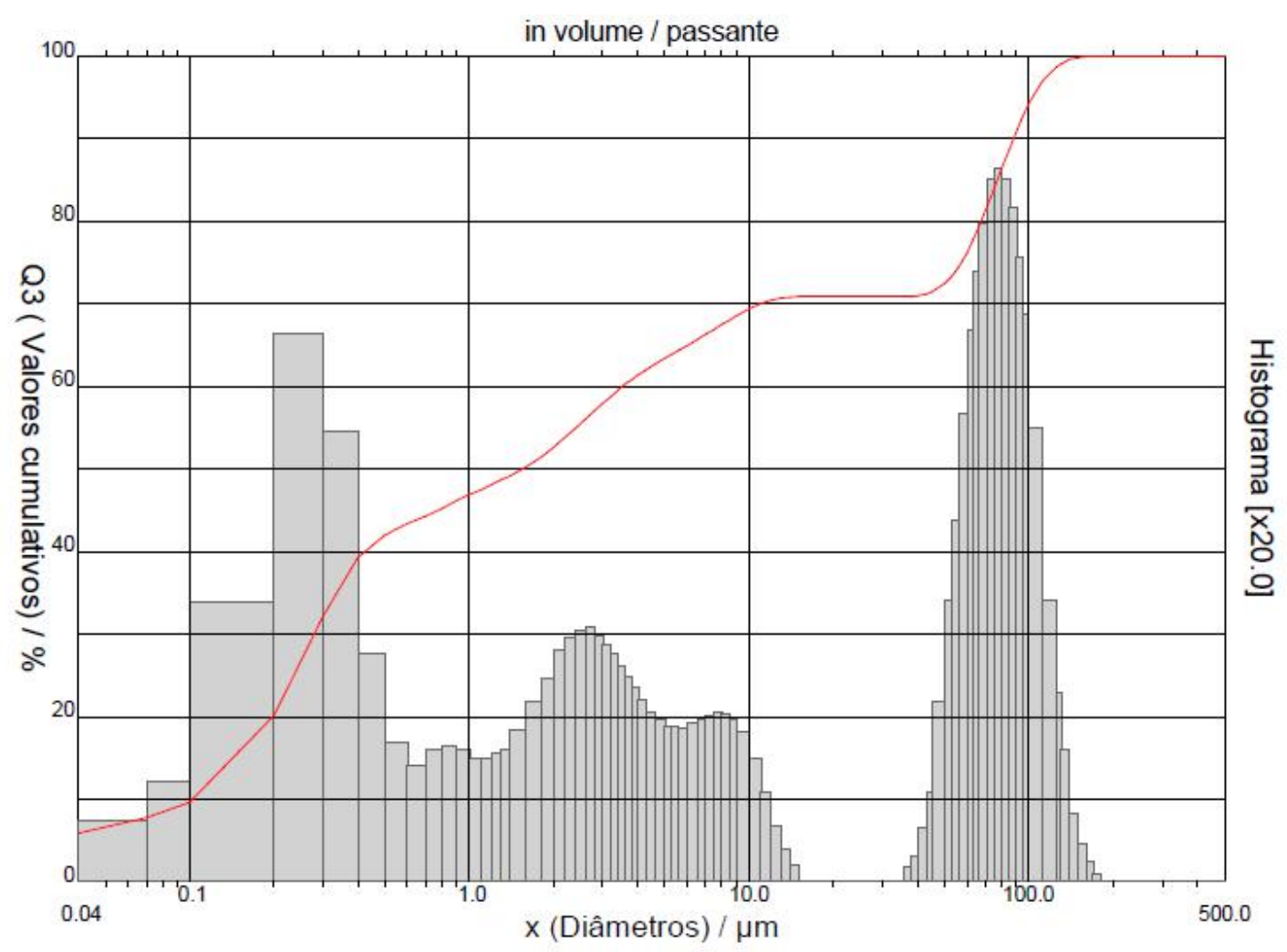

Figura 4 - Histograma obtido da dispersão do tamanho de partículas

O tamanho médio das partículas encontradas foi de $24,89 \mu \mathrm{m}$, o que não está congruente com a análise por microscopia eletrônica. Vale ressaltar que, este ensaio não foi realizado com as nanoesferas centrifugadas, mas sim com a solução obtida após a síntese, o que justifica a discrepância desses valores. No entanto, é importante mencionar que o histograma também apresenta tres faixas distintas para os tamanhos um tem seu máximo em $0,30 \mu \mathrm{m}(300 \mathrm{~nm})$, outro em $3 \mu \mathrm{m}(3000 \mathrm{~nm})$ e o terceiro em $100 \mu \mathrm{m}(10000 \mathrm{~nm})$.

Segundo os dados obtidos, $10 \%$ das partículas tem até $0,10 \mu \mathrm{m}$, a faixa de $50 \%$ está em 1,55 $\mu \mathrm{m}$ e $90 \%$ das partículas estão até $88,20 \mu \mathrm{m}$. Portanto, segundo essa medição, cerca de $30 \%$ 
das nanoesferas estão na faixa ótima de tamanho para aplicação em sistema biológico que é abaixo de 0,2 $\mu \mathrm{m}$ de acordo com Shubayev e colaboradores (2009).

Comparando-se os dados obtidos por granulometria a laser e as imagens ao MEV é possivel sugerir que o procedimento adotado para a separação das esferas de PS através de solução com isopropanol e centrigugação gerou uma separação por faixa de tamanhos. Assim o material recolhido e analisado ao MEV já apresentava uma separação prévia das partículas maiores por decantação.

\section{CONCLUSÃO}

A síntese de nanoesferas de poliestireno por via livre de emulsificante se mostrou eficaz. A suspensão de nanoesferas se mostrou estável, uma vez que depois de realizada se manteve inalterada por várias semanas. Além disso, o resultado do MEV mostrou que de fato o produto final era o desejado e conjunto com os resultados da granulometria, que mostrou o tamanho certo das nanoesferas, provou o sucesso na execução da síntese. Ressalta-se, porém, que por se tratar de um material nanométrico houve uma dificuldade para que decantasse e pudessem se realizar os ensaios. O próximo passo para o avanço dessa linha de pesquisa refere-se ao encapsulamento das nanopartículas magnéticas para o tratamento tumoral. Antes porém, se fazem necessárias maiores investigações na etapa de síntese, visando a menor distribuição de tamanho de partículas e na separação por centrifugação privilegiando a obtenção de partículas menores de $200 \mathrm{~nm}$.

\section{REFERÊNCIAS}

ALEXIS, Frank et al. New frontiers in nanotechnology for cancer treatment. Urologic Oncology: Seminars and Original Investigations, [s.1.], v. 26, n. 1, p.74-85, jan. 2008. Elsevier BV.

BHATTACHARYA, R; MUKHERJEE, P. Biological properties of "naked" metal nanoparticles. Advanced Drug Delivery Reviews, [s.1.], v. 60, n. 11, p.1289-1306, 17 ago. 2008. Elsevier BV.

BOURGEAT-LAMI, E.; LANG, J. Encapsulation of Inorganic Particles by Dispersion Polymerization in Polar Media. Journal of Colloid and Interface Science, v. 197, p. 293-308, 1998.

DUMONT, Marcello Rosa. Desenvolvimento de Alumina- $\gamma$ mesoporosa dopada com céria e zircônia pelo processo sol-gel para aplicações em catalisadores automotivos. 2016. 177 f. Tese (Doutorado) - Engenharia Metalúrgica, Materiais e de Minas, UFMG, Belo Horizonte, 2016.

FARIDI-MAJIDI, R.; SHARIFI-SANJANI, N.; AGEND, F. Encapsulation of magnetic nanoparticles with polystyrene via emulsifier-free miniemulsion polymerization. Thin Solid Films, v. 515, p. 368-374, 2006.

FAROKHZAD, O; LANGER, R. Nanomedicine: Developing smarter therapeutic and diagnostic modalities. Advanced Drug Delivery Reviews, [s.1.], v. 58, n. 14, p.14561459, 1 dez. 2006. Elsevier BV. 
FIGUEROLA, A. et al. From iron oxide nanoparticles towards advanced iron-based inorganic materials designed for biomedical applications. Pharmacological Research, v. 62, p. 126-143, 2010.

GIUSTINI, A. J. et al. Magnetic nanoparticle hyperthermia in cancer treatment. Nano LIFE, v. 1, n. 1\&amp;2, p. 17-32, 2010.

HE, Guangwei; PAN, Qinmin. Synthesis of Polystyrene and Polystyrene/Poly(methyl methacrylate) Nanoparticles. Macromolecular Rapid Communications, [s.1.], v. 25, n. 17, p.1545-1548, 9 set. 2004. Wiley-Blackwell.

HUANG, H.-C. et al. Inorganic nanoparticles for cancer imaging and therapy. Journal of Controlled Release, v. 155, p. 344-357, 2011.

LADJ, Rachid et al. Polymer encapsulation of inorganic nanoparticles for biomedical applications. International Journal Of Pharmaceutics, [s.1.], v. 458, n. 1, p.230-241, dez. 2013. Elsevier BV.

LADJA, R. et al. Polymer encapsulation of inorganic nanoparticles for biomedical applications. International Journal of Pharmaceutics, v. 458, p. 230-251, 2013.

LANDFESTER, Katharina. Miniemulsion Polymerization and the Structure of Polymer and Hybrid Nanoparticles. Angewandte Chemie International Edition, [s.1.], v. 48, n. 25, p.4488-4507, 8 jun. 2009. Wiley-Blackwell.

LIANG, C. Y.; KRIMM, S. Infrared Spectra of High Polymers. Journal Of Polymer Science, p.241-254,1958.

LOIOLA, Adonay R. et al. Synthesis and characterization of hierarchical porous materials incorporating a cubic mesoporous phase. Journal of Materials Chemistry, [s.1.], v. 18, n. 41, p.4985-4993, set. 2008. Royal Society of Chemistry (RSC).

LUO, Bin et al. Multi-Functional Thermosensitive Composite Microspheres with High Magnetic Susceptibility Based on Magnetite Colloidal Nanoparticle Clusters. Langmuir, [s.1.], v. 26, n. 3, p.1674-1679, 2 fev. 2010. American Chemical Society (ACS). (LOU et al. 2010)

MACHADO, Fabricio; LIMA, Enrique L.; PINTO, José Carlos. Uma revisão sobre os processos de polimerização em suspensão. Polímeros, [s.1.], v. 17, n. 2, jun. 2007. FapUNIFESP (SciELO).

MAHMOUDI, M. et al. Superparamagnetic iron oxide nanoparticles (SPIONs): Development, surface modification and applications in chemotherapy. Advanced Drug Delivery Reviews, v. 63, p. 24-46, 2011.

MISRA, R.; ACHARYA, S.; SAHOO, S. K. Cancer nanotechnology: application of nanotechnology in cancer therapy. Drug discovery today, v. 15, p. 842-850, 2010.

NANDIYANTO, Asep Bayu Dani et al. Mesopore-free silica shell with nanometer-scale thickness-controllable on cationic polystyrene core. Journal Of Colloid And Interface Science, [s.1.], v. 389, n. 1, p.134-146, jan. 2013. Elsevier BV. http://dx.doi.org/10.1016/j.jcis.2012.08.054.

NANDIYANTO, Asep Bayu Dani et al. Synthesis of additive-free cationic polystyrene particles with controllable size for hollow template applications. Colloids And Surfaces A: Physicochemical and Engineering Aspects, [s.1.], v. 396, p.96-105, fev. 2012. Elsevier BV.

RAMOS, J.; FORCADA, J. Surfactant-Free Miniemulsion Polymerization as a Simple Synthetic Route to a Successful Encapsulation of Magnetite Nanoparticles. Langmuir, v. 27, p. 7222-7230, 2011. 
RIEGER, J.. THE GLASS TRANSITION TEMPERATURE OF POLYSTYRENE: Results of a round robin test. Journal Of Thermal Analysis, Ludwigshafen, v. 46, n. 1, p.965972, jan. 1996.

SEM, T. et al. Synthesis and Characterization of Hierachically Ordered Porous Silica Materials. Chemistry of Materials, 16, 2004. 2044-2054.

SHUBAYEV, V. I.; II, T. R. P.; JIN, S. Magnetic nanoparticles for theragnostics. Advanced Drug Delivery Reviews, v. 61, p. 467-477, 2009.

SHUBAYEV, Veronica I.; PISANIC, Thomas R.; JIN, Sungho. Magnetic nanoparticles for theragnostics. Advanced Drug Delivery Reviews, [s.1.], v. 61, n. 6, p.467-477, jun. 2009. Elsevier BV.

SIEGEL, R.; NAISHADHAM, D.; JEMAL, A. Cancer Statistics, 2012. CA: A Cancer Journal for Clinicians, v. 62, p. 10-29, 2012.

WIBAWA, Pratama Jujur et al. Design, Preparation and Characterization of Polystyrene Nanospheres Based-Porous Structure towards UV-Vis and Infrared Light Absorption. Physics Procedia, [s.1.], v. 22, p.524-531, 2011. Elsevier BV. 